\title{
ULTRATHIN LOUDSPEAKERS FROM ALD
}

\author{
Joseph J. Brown", Nathan C. Moore, Omkar D. Supekar, and Victor M. Bright \\ Department of Mechanical Engineering, University of Colorado, Boulder, Colorado, USA
}

\begin{abstract}
Arrays of suspended microbridges (Figures 1 and 2) with sizes as small as $17 \mu \mathrm{m} \times 1 \mu \mathrm{m}$ have been fabricated and demonstrated to produce audible sound using the thermoacoustic effect. The microbridges were fabricated by atomic layer deposition (ALD) of $6.5 \mathrm{~nm} \mathrm{Al}_{2} \mathrm{O}_{3}$ and $19.3 \mathrm{~nm}$ tungsten on sacrificial polyimide [1], with ALD performed at $130{ }^{\circ} \mathrm{C}$ and patterned with standard photolithography. Loudspeaker sensitivity of these first-generation devices is $\sim 29 \mathrm{~dB}$ SPL/1 mW. These devices demonstrate industrially feasible nanofabrication of thermoacoustic transducers and a sound production mechanism pertinent to submicron-scale device engineering.
\end{abstract}

\section{INTRODUCTION}

The development of nanomaterials and nanoscale engineering has enabled new capabilities in nanoscale thermal engineering. These have, in turn, led to a resurgence of interest in thermoacoustic sound production. In this transduction mechanism, Joule heating creates temperature fluctuations in a solid material, heat conduction generates coupled temperature and pressure fluctuations in the surrounding fluid, and the pressure fluctuations propagate away from the device as sound waves. This transduction mechanism was first explored in the early $20^{\text {th }}$ century [2]. Work on porous Si in 1999 [3] and CNTs in 2008 [4] initiated the wave of recent interest in thermoacoustic transducers. In addition to porous $\mathrm{Si}$ and CNTs [4]-[9], thermoacoustic loudspeakers have been demonstrated from nanowire arrays [10][13], and graphene [14]-[18]. This prior work has suggested the following principles to maximize nanomaterial sound production:

(1) Minimize substrate losses by suspending devices [11], [17].

(2) Minimize the areal heat capacity of the transducer [16], [17]. The devices here have $0.18 \mathrm{~J} \mathrm{~m}^{-2} \mathrm{~K}^{-1}$ (calculated from Refs. [19], [20]), lower than any sample in Ref. [21].

(3) Maximize or concentrate heat flux $q_{0}\left[\mathrm{~J} \mathrm{~m}^{-2}\right]$ from the transducer (see Eqs. 1 and 2, below) [5], [12].

(4) Maximize the heat transfer to the surrounding fluid, such as by maximizing the transducer surface area, and distribute power evenly through a sample [21].

Devices using ultrathin $(<50 \mathrm{~nm})$ films produced by ALD allow engineering according to these principles. In particular, ALD provides control of heat capacity and thermal conductivity. Furthermore, ALD is readily integrated in manufacturing, having already been integrated into wafer-scale CMOS processing. It may be expanded to continuous roll-to-roll production, adapted for flexible substrates, and used to produce many materials including insulators and metals. Resolution is limited only by lithography.

\section{Thermoacoustic Physics}

Thermoacoustic loudspeakers operate at low voltages, providing an advantage over piezoelectric, electrostatic, and other capacitive acoustic transducers. Very good (85-100 dB SPL in air, $200 \mathrm{~dB}$ SPL for encapsulated devices in water) sound production has been measured from thermoacoustic devices [9], [21]. For most audio frequencies, the efficiency $\eta$ (sound power out/electrical power in) is typically $\sim 10^{-6}$ to $10^{-5}$ at best [5], [10][13], [17], [21]. In auditory frequencies these devices typically have a frequency-dependent behavior of sound pressure and

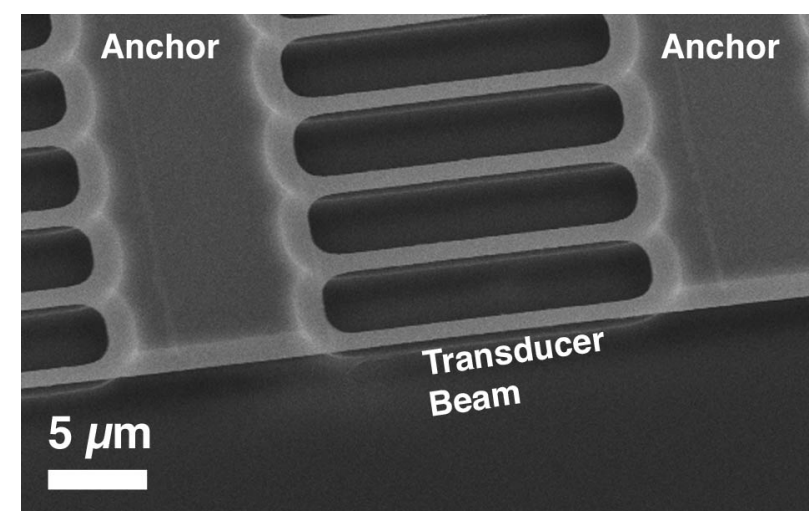

Figure 1: SEM image of suspended microbridges used for thermoacoustic sound generation. Beams are $17 \mu \mathrm{m}$ long, $1 \mu \mathrm{m}$ wide, $25.8 \mathrm{~nm}$ thick and suspended $3.5 \mu \mathrm{m}$ above the substrate. Anchor regions provide mechanical connection to the substrate.

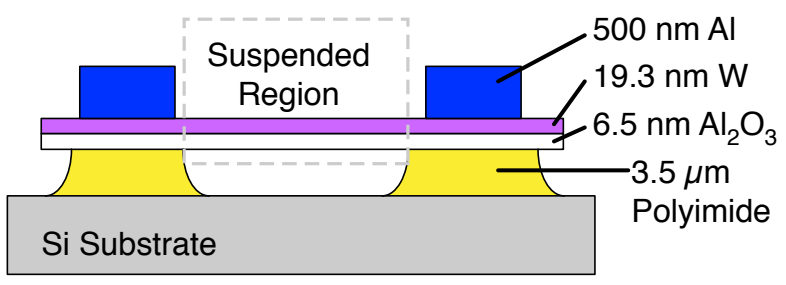

Figure 2: Cross-section diagram of the fabricated devices.

efficiency, increasing with frequency $f$ (Eq. 1) until saturating at some maximum level (Eq. 2) [3], [5], [12], [22]. Here, $\rho_{g}$ is the density of air $\left(\sim 1.2 \mathrm{~kg} / \mathrm{m}^{3}\right), v_{g}$ is the isothermal speed of sound in air $(\sim 340 \mathrm{~m} / \mathrm{s}), c_{p}$ is the specific heat of air $\left(\sim 1007 \mathrm{~J} \mathrm{~kg}^{-1} \mathrm{~K}^{-1}\right), T_{0}$ is ambient temperature $(\sim 300 \mathrm{~K})$, and $A$ is the area of a transducer.

$$
\begin{aligned}
& \eta=\frac{\pi f^{2}}{4 \rho_{g} v_{g} c_{p}^{2} T_{0}^{2}} q_{0} A \\
& \eta=\frac{q_{0} v_{g}}{2 \rho_{g} c_{p}^{2} T_{0}^{2}}
\end{aligned}
$$

Under certain conditions, i.e. plane wave production and $\omega>>$ $\alpha_{s} / 2 L_{s}^{2}$ (where $\omega=2 \pi f, \alpha_{s}$ is the thermal diffusivity of the transducer film, and $L_{s}$ is the distance through which heat propagates to a substrate), the frequency dependence of Eq. 1 may be mitigated, resulting in a relatively flat frequency response [22]. In encapsulated systems, other behavior may emerge such as $f^{-3 / 2}$ dependence [2] and mechanical resonance [5]. Optimization of these transducers is a balance among efficiency, electrical power consumption, sound output, manufacturability, and packaging.

\section{THERMOACOUSTIC MODEL}

The development of practical models for thermoacoustics is an area of ongoing inquiry and optimization [21]. The fundamental physical problem is expressed by coupled temperature 
and pressure field equations [12], [23]. Most models take a distributed approach or ignore some of the relevant conduction effects in the transducer materials. With some simplification [5], thermoacoustic sound generation can be interpreted as a source driving a temperature wave, which drives a propagating pressure wave. The thermal wave decays rapidly, with diffusion length $\mu_{i}$ $(100 \mathrm{~Hz})=(\alpha / \pi f)=268 \mu \mathrm{m}$ and $\left.\mu_{i}(100 \mathrm{kHz})=8.46 \mu \mathrm{m}\right)$ [23], where $\alpha$ is thermal diffusivity of air $\left(2.25 \times 10^{-5} \mathrm{~m}^{2} / \mathrm{s}\right)$.

Suspended thermoacoustic bridges of finite length have not been thoroughly considered in literature, with the closest models assuming infinite length beams [12]. While this assumption allows assessment of the upper bounds of sound production efficiency (Eqs. 1-3), it ignores effects of thermal conduction within finite beams on the output frequency spectrum. The thermal diffusivity calculated for ALD W beam material is $\alpha_{s}=2.25 \times 10^{-6} \mathrm{~m}^{2} \mathrm{~s}^{-1}$ [19], [20]. Combined with typical thermal conduction lengths of suspended microbridges, the criterion $\omega>\alpha_{s} / 2 L_{s}{ }^{2}$ may be satisfied for low frequencies, such as $20 \mathrm{~Hz}$ for $600 \mu \mathrm{m}$ beams. This suggests that models of thermoacoustic emission from nanoscale beams should account for heat flux through the beams in addition to emitted power. This is accomplished below in a new model for heat flow from thermoacoustic transducers that is distinct from previous models in that (1) it accounts for heat conduction and capacitance in microbridges of finite length, and (2) it does so using a lumped element approach.

\section{Lumped Element Model for Microbridge Sound Production}

In each transducer beam, energy production from Joule heating is balanced (Eq. 3) by emission of a thermal wave from the top surface of the beam (Eq. 4), heat conduction and storage in the beam (Eq. 5), and, from the bottom surface, both emission of a thermal wave and conduction through the air to the underlying fabrication substrate (Eq. 6). Here each $\dot{Q}$ term is a lumped heat power flow and harmonic analysis is used to find solutions to these equations, which is a common approach in thermoacoustic analysis. Heat flow from the top surface is given by the 1D thermal wave output, Eq. 4, wherein $\kappa$ is thermal conductivity of air $\left(0.0263 \mathrm{~W} \mathrm{~m}^{-1} \mathrm{~K}^{-1}\right), T_{A C}$ is the oscillating component of the beam temperature, $L_{B}$ is the beam length, $w_{B}$ is the beam width, and $k_{1}$ is the thermal wavenumber, given by $k_{1}=(j \omega / \alpha)^{1 / 2}$. Heat loss in the beam is described by Eq. 5, where the thermal resistance $R_{\text {beam }}=\left(L_{B} / 4\right) /\left(\kappa_{f} \delta w_{B}\right)$ and thermal capacitance $C_{\text {beam }}=\rho_{f} c_{f} w_{B}$ $L_{B} / 2$. Film thickness is $\delta$, film density is $\rho_{f}$, specific heat of the film is $c_{f}$, and thermal conductivity of the film is $\kappa_{f}$. Input power supplied to the transducer beam is a modeled as $P_{A C}=P_{0} \mathrm{e}^{-j \omega t}$. In reality $\mathrm{AC}$ Joule heating power can have several frequency components. The power flowing from the bottom of the beam, Eq. 6 , is a combination of a thermal wave and heat conduction to the substrate, with $R_{\text {gap }}$ approximated as $R_{\text {gap }}=g /\left(\kappa_{f} L_{B} w_{B}\right)$, where $g$ is the gap distance between the beam and substrate.

$$
\begin{aligned}
& \dot{Q}_{\text {in }}=\dot{Q}_{\text {top }}+\dot{Q}_{\text {bottom }}+\dot{Q}_{\text {beam }} \\
& \dot{Q}_{\text {top }}=-j \kappa k_{1} T_{A C} L_{B} w_{B} \\
& \dot{Q}_{\text {beam }}=T_{A C} R_{\text {beam }}^{-1}-j \omega C_{\text {beam }} T_{A C} e^{-j \omega t} \\
& \dot{Q}_{\text {bottom }}=-j \kappa k_{1} T_{A C} L_{B} w_{B}+T_{A C} R_{\text {gap }}^{-1}
\end{aligned}
$$

Rewriting Eq. 3 using Eqs. 4-6 yields the system model Eq. 7. If $P_{A C}$ is given, this allows calculation of $T_{A C}$, "piston velocity" $u$, rms sound pressure $p_{r m s}$, and sound production efficiency $\eta$, Eqs.
9-12. Calculation of $u$ uses the continuity and ideal gas equations [5], and assumes sound generated from each beam is distributed across the width $d_{n}$ that is allocated to each beam in an array $\left(d_{n}=\right.$ beam width + beam separation).

$$
\begin{gathered}
T_{A C}=P_{A C}\left[-2 j \kappa k_{1} L_{B} w_{B}+R_{\text {beam }}^{-1}-j \omega C_{\text {beam }}+R_{g a p}^{-1}\right]^{-1} \\
u=2\left(\frac{\omega}{k_{1}}\right)\left(\frac{T_{A C}}{T_{0}}\right)\left(\frac{w_{B}}{d_{n}}\right) \\
p_{r m s}=\rho_{g} v_{g} \frac{|u|}{\sqrt{2}} \\
\eta=\frac{\left(p_{r m s}^{2} / \rho_{g} v_{g}\right)}{\left(\dot{Q}_{0} / L_{B} d_{n}\right)}=\frac{p_{r m s}^{2} L_{B} d_{n}}{P_{A C} \rho_{g} v_{g}}
\end{gathered}
$$

Although the $P_{A C}$ appears in the denominator in Eq. 10, when Eq. 7 is carried through Eqs. $8-10$, the result is that $P_{A C}$ is in the numerator and $\eta \propto P_{A C}$, similar to Eqs. 1 and 2. If the losses of Eqs. 5 and 6 are omitted, Eq. 2 can be recovered from Eqs. 7-10. The sound pressure level (SPL) from one device is related to $\eta$ using $p_{r m s} 2$, given SPL $=10 \log _{10}\left(p_{r m s}{ }^{2} / p_{0}^{2}\right)$, with reference pressure $p_{0}=2 \times 10^{-5} \mathrm{~Pa}$. Figure 3 examines $\eta$ and SPL under several conditions. When there are no losses through the beam or conduction through air, the sound output is near the maximum of Eq. 2. Losses in the beam maintain a relatively flat output. Conduction through the air to the substrate significantly decreases the low frequency output. In Eqs. 7-10, the only mechanisms to improve $\eta$ are to increase $P_{A C}$, increase $R_{\text {beam }}$ and $R_{\text {gap }}$, decrease $C_{\text {beam }}$, and to select beam size and spacing to maximize $u$ and $T_{A C}$.

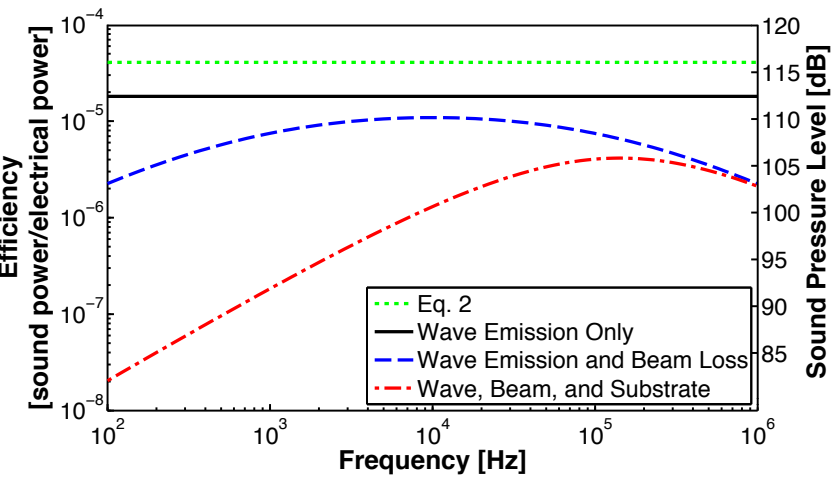

Figure 3: Near-field model result for applied power of $1 \mathrm{~W} / \mathrm{cm}^{2}$ (device total $0.195 \mathrm{~W}$ ) from one of the 17- $\mu \mathrm{m}$ microbridge transducers described below. This figure compares the effect on sound production efficiency $\eta$ of the different loss terms on the right-hand side of Eq. 7.

\section{EXPERIMENT}

\section{Device Design and Fabrication}

Microbridge arrays were designed such that series and parallel electrical connections among the microbridges would allow a total array electrical resistance of $10-20 \Omega$. A $500 \mathrm{~nm} \mathrm{Al} \mathrm{layer} \mathrm{was}$ deposited on the $\mathrm{W}$ film in order to distribute electrical power throughout the array. One design used 12,000 microbridges 600 $\mu \mathrm{m} \times 2 \mu \mathrm{m}$ in size. A second variation used 576,000 microbridges $17 \mu \mathrm{m} \times 1 \mu \mathrm{m}$ in size. Devices were fabricated on $15 \mathrm{~mm} \times 15$ $\mathrm{mm} \mathrm{Si}$ chips as described in Figure 4. 

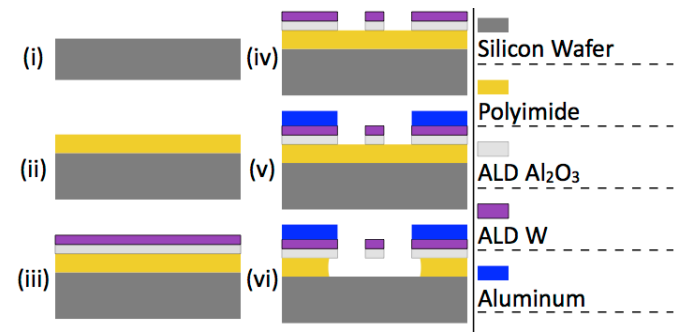

Figure 4: Fabrication process. (i) Provide a silicon wafer substrate. (ii) Spin-coat a $3.5 \mu \mathrm{m}$ polyimide layer. (iii) Perform ALD of alumina $(6.5 \mathrm{~nm})$ and tungsten $(19.3 \mathrm{~nm})$ at $130^{\circ} \mathrm{C}$. (iv) Pattern the ALD layers using $\mathrm{O}_{2} / \mathrm{CF}_{4}$ RIE. (v) Deposit and pattern $500 \mathrm{~nm}$ of aluminum. (vi) Release the microbridge structures using an isotropic $\mathrm{O}_{2}$ plasma etch.

\section{Acoustic Characterization}

An anechoic chamber with 18 " $\times 18$ " base and 15 " height was built using with $\geq 6$ " of open cell foam surrounding all sides, of which 3" were pyramid-shaped. Current delivered to each transducer array was determined by monitoring the voltage across a $1-\Omega$ resistor in series with the device. Sound waves were recorded (Figure 5) by a calibrated microphone (Dayton Audio EMM-6). Data were collected by an NI USB-6259 with $100 \mathrm{kHz}$ sampling rate, and processed with LabVIEW and MATLAB. Acoustic performance was measured from device arrays with $0-$ $10 \mathrm{~V}$ supplied by an Agilent 33250A function generator, or unbiased voltage from an audio amplifier (Dayton APA150).

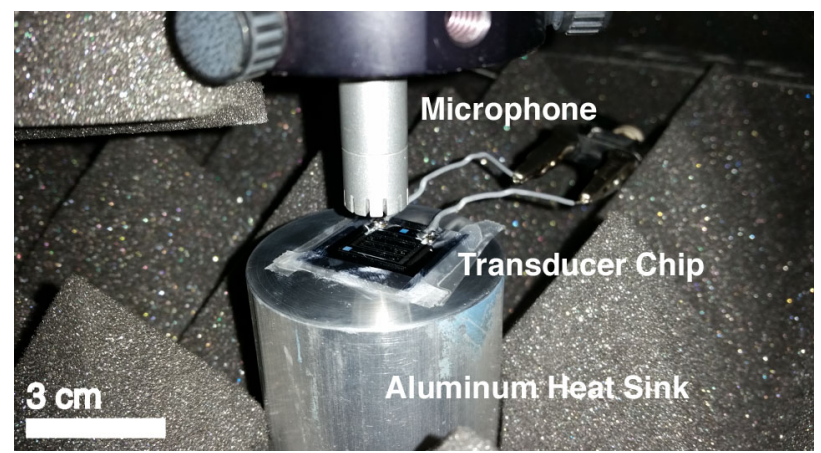

Figure 5: Near-field experimental setup in anechoic chamber.

\section{RESULTS AND DISCUSSION}

Under a linear chirp (frequency varies linearly with time), over $50 \mathrm{~dB}$ SPL was generated at frequencies above $10 \mathrm{kHz}$ (Figure 6). Measured device arrays with $0.0838 \mathrm{~W}$ input power displayed $48.5 \mathrm{~dB}$ sound pressure level at $1 \mathrm{~cm}$ distance (Figure 7). This is much less than the $\sim 92 \mathrm{~dB}$ SPL that the model predicts for this power input. Spherical wave propagation, losses in electrical connections, and other device impedances resulting from the experimental setup account for a portion of this discrepancy and may accommodated with further optimization. For instance, device resistance was higher than designed, on the order of $40-$ $100 \Omega$, largely due to solder connections. Efficiency of these firstgeneration devices ( $\eta=\sim 10^{-10}$, Figure 7 ) is comparable to other thermoacoustic nanomaterial devices but much less than commercial headphones $\left(\sim 100 \mathrm{~dB}\right.$ per $1 \mathrm{~mW}$, or $\sim 10^{-3}$ for a device with $1 \mathrm{~cm}^{2}$ area). Even with no beam and substrate losses, maximum $T_{A C}$ at $1 \mathrm{~W} / \mathrm{cm}^{2}$ input is $92 \mathrm{~K}$. Clearly the transducers can be driven with much higher power. The microbridge arrays displayed good fidelity, with relatively flat spectral response across the audible range, Figure 8 . This may be expected because electrical impedance is due primarily to resistivity, providing an advantage over conventional electromagnetic voice-coil loudspeakers, which have resonance peaks due to inductance and mechanical dynamics.

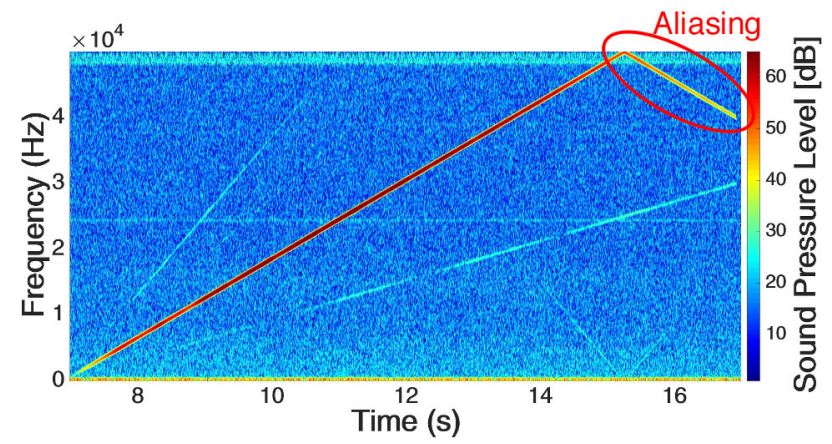

Figure 6: Spectrogram of time-varying sound output. Electrical input: $50 \mathrm{~Hz}-30 \mathrm{kHz}$ unbiased linear chirp. Chip output: $100 \mathrm{~Hz}$ - $60 \mathrm{kHz}$ due to frequency doubling from Joule heating. Measured at $5 \mathrm{~cm}$ microphone distance (add $14 \mathrm{~dB}$ for $1 \mathrm{~cm}$ equivalent).

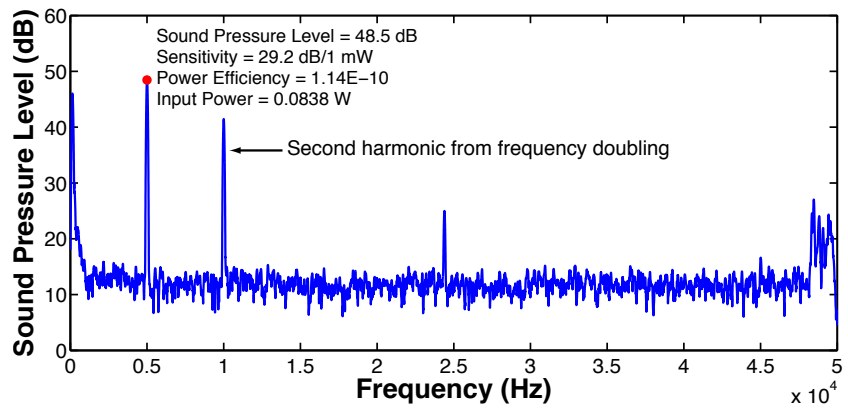

Figure 7: Single tone frequency response measured at $1 \mathrm{~cm}$ microphone distance, $0-10 \mathrm{~V}, 5 \mathrm{kHz}$ input.

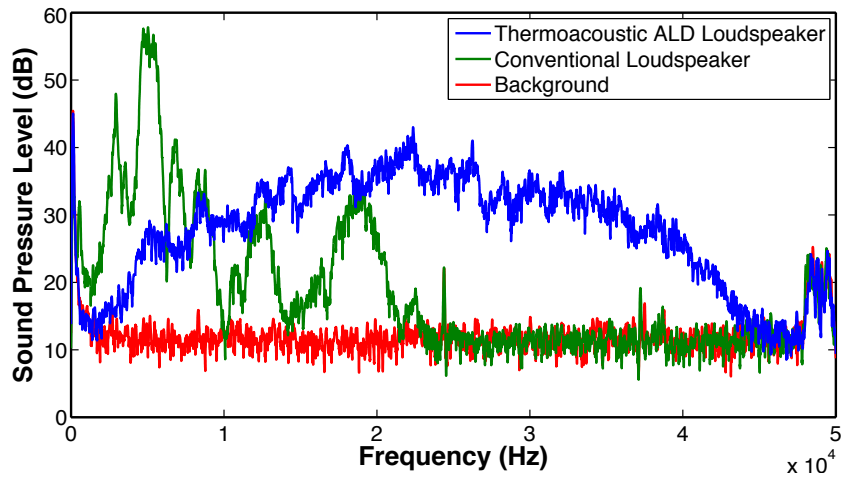

Figure 8: Spectral output for thermoacoustic devices, compared with a conventional electromagnetic loudspeaker $37 \mathrm{~mm}$ in diameter. Sound output spectra in response to white noise electrical input. The conventional speaker displays resonant peaks in the $5-25 \mathrm{kHz}$ range. The white noise was supplied to the thermoacoustic device $(R=83.8 \Omega)$ as an electrical current of $0.103 A_{\text {rms }}$, with $0.88 \mathrm{~W}$ total. The thermoacoustic device shows relatively broad frequency response, peaking around $25 \mathrm{kHz}$. 


\section{CONCLUSIONS}

Planar microbridge array loudspeakers formed using ALD and microfabrication were produced and demonstrated to operate in the audible range, with frequency response clearly distinct from an electromagnetic loudspeaker. Transducers consisted of suspended $25.8 \mathrm{~nm}$ thick microbridges subject to Joule heating, producing sound by means of the thermoacoustic effect. This transduction mechanism provides a compact means of generating pressure fluctuations in a fluid, and can integrate readily with other microdevices. The operation of arrays of devices provides resilience against failure of individual microbridges, and demonstrates a macroscale effect from large-scale parallelization of microdevices. Analysis of the thermoacoustic transducers indicates that geometry and materials selection play critical roles in optimization of the efficiency and frequency response.

Although the efficiency of these devices in the audible range is below that of electromagnetic transducers, the frequency spectrum can achieve relatively flat response. The devices are scalable to both large area and nanoscale operation. Modeling predicts near-field sound pressure levels of $>90 \mathrm{~dB}$ from thermoacoustic microbridges. Further nanoengineering may allow the devices to reach their ultimate limits, through reduction of heat capacity, and improvement of substrate reflectance. Far field sound levels will improve with use of larger arrays. Device encapsulation may provide an additional mechanism to improve efficiency of audible sound production.

\section{ACKNOWLEDGEMENTS}

Dr. N.T. Eigenfeld, J.C. Gertsch, and Prof. S.M. George are acknowledged for helpful conversations, collaboration on the fabrication process, and assistance with ALD processing. Dr. S.E. Fick of NIST-Gaithersburg is acknowledged for helpful conversations regarding audio characterization.

\section{REFERENCES}

[1] N.T. Eigenfeld, J.M. Gray, J.J. Brown, G.D. Skidmore, S.M. George, and V.M. Bright, "Ultra-Thin 3D Nano-Devices from Atomic Layer Deposition on Polyimide", Adv. Mater., 26, 3962 (2014).

[2] H.D. Arnold and I.B. Crandall, "The Thermophone as a Precision Source of Sound", Phys. Rev., 10, 22 (1917).

[3] H. Shinoda, T. Nakajima, K. Ueno, and N. Koshida, "Thermally Induced Ultrasonic Emission from Porous Silicon", Nature, 400, 853 (1999).

[4] L. Xiao, Z. Chen, C. Feng, L. Liu, Z.Q. Bai, Y. Wang, L. Qian, Y. Zhang, Q. Li, K. Jiang, and S. Fan, "Flexible, Stretchable, Transparent Carbon Nanotube Thin Film Loudspeakers", Nano Lett., 8, 4539 (2008).

[5] A.E. Aliev, Y.N. Gartstein, and R.H. Baughman, "Increasing the Efficiency of Thermoacoustic Carbon Nanotube Sound Projectors", Nanotechnology, 24, 235501 (2013).

[6] Y. Wei, X. Lin, K. Jiang, P. Liu, Q. Li, and S. Fan, "Thermoacoustic Chips with Carbon Nanotube Thin Yarn Arrays", Nano Lett., 13, 4795 (2013).

[7] A.E. Aliev, N.K. Mayo, R.H. Baughman, D. Avirovik, S. Priya, M.R. Zarnetske, and J.B. Blottman, "Thermal Management of Thermoacoustic Sound Projectors Using a Free-Standing Carbon Nanotube Aerogel Sheet as a Heat Source", Nanotechnology, 25, 405704 (2014).

[8] K. Suzuki, S. Sakakibara, M. Okada, Y. Neo, H. Mimura, Y. Inoue, and T. Murata, "Study of Carbon-Nanotube Web Thermoacoustic Loud Speakers," Jpn. J. Appl. Phys., 50, 01BJ10 (2011).
[9] S.S. Asadzadeh, A. Moosavi, C. Huynh, and O. Saleki, "Thermo Acoustic Study of Carbon Nanotubes in Near and Far Field: Theory, Simulation, and Experiment", J. Appl. Phys., 117, 095101 (2015).

[10] H. Tian, D. Xie, Y. Yang, T.L. Ren, Y.X. Lin, Y. Chen, Y.F. Wang, C.J. Zhou, P.G. Peng, L.G. Wang, and L.T. Liu, "Flexible, Ultrathin, and Transparent Sound-Emitting Devices Using Silver Nanowires Film", Appl. Phys. Lett., 99, 253507 (2011).

[11] R. Dutta, B. Albee, W.E. Van Der Veer, T. Harville, K.C. Donovan, D. Papamoschou, and R.M. Penner, "Gold Nanowire Thermophones”, J. Phys. Chem. C, 118, 29101 (2014).

[12] V. Vesterinen, A.O. Niskanen, J. Hassel, and P. Helistö, "Fundamental Efficiency of Nanothermophones: Modeling and Experiments," Nano Lett., 10, 5020 (2010).

[13] A.O. Niskanen, J. Hassel, M. Tikander, P. Maijala, L. Grönberg, and P. Helistö, "Suspended Metal Wire Array as a Thermoacoustic Sound Source”, Appl. Phys. Lett., 95, 2012 (2009).

[14] H. Tian, T.L. Ren, D. Xie, Y.F. Wang, C.J. Zhou, T.T. Feng, D. Fu, Y. Yang, P.G. Peng, L.G. Wang, and L.T. Liu, "Graphene-on-Paper Sound Source Devices", ACS Nano, 5, 4878 (2011).

[15] H. Tian, C. Li, M.A. Mohammad, Y.L. Cui, W.T. Mi, Y. Yang, D. Xie, and T.L. Ren, "Graphene Earphones: Entertainment for Both Humans and Animals," ACS Nano, 8, 5883 (2014).

[16] H. Tian, D. Xie, Y. Yang, T.-L. Ren, Y.-F. Wang, C.-J. Zhou, P.-G. Peng, L.-G. Wang, and L.-T. Liu, "Single-layer Graphene Sound-Emitting Devices: Experiments and Modeling", Nanoscale, 4, 2272 (2012).

[17] J.W. Suk, K. Kirk, Y. Hao, N.A. Hall, and R.S. Ruoff, "Thermoacoustic Sound Generation from Monolayer Graphene for Transparent and Flexible Sound Sources", Adv. Mater., 24, 6342 (2012).

[18] H. Tian, Y. Yang, D. Xie, J. Ge, and T.-L. Ren, “A reduced Graphene Oxide Sound-Emitting Device: a New Use for Joule Heating", RSC Adv., 3, 17672 (2013).

[19] N.T. Eigenfeld, J.M. Gray, J.C. Gertsch, G.D. Skidmore, S.M. George, and V.M. Bright, "Specific Heat Capacity of UltraThin Atomic Layer Deposition Nanobridges for Microbolometers", Proceedings of 2015 Transducers - the 2015 18th International Conference on Solid-State Sensors, Actuators and Microsystems (TRANSDUCERS), Anchorage, AK, 6/21-25/15, IEEE, New York (2015), pp. 1385-1388.

[20] N.T. Eigenfeld, J.C. Gertsch, G.D. Skidmore, S.M. George, and V.M. Bright, "Electrical and Thermal Conduction in Ultra-thin Freestanding Atomic Layer Deposited W Nanobridges", Nanoscale, 7, 17923 (2015).

[21] A.E. Aliev, N.K. Mayo, M. Jung De Andrade, R.O. Robles, S. Fang, R.H. Baughman, M. Zhang, Y. Chen, J.A. Lee, and S.J. Kim, "Alternative Nanostructures for Thermophones", ACS Nano, 9, 4743 (2015).

[22] H. Hu, Y. Wang, and Z. Wang, "Wideband Flat Frequency Response of Thermo-acoustic Emission", J. Phys. D. Appl. Phys., 45, 345401 (2012).

[23] F.A. McDonald and G.C. Wetsel, Jr., "Generalized Theory of the Photoacoustic Effect”, J. Appl. Phys., 49, 2313 (1978).

\section{CONTACT}

*J.J. Brown, tel: +1-303-735-8054; joseph.j.brown@colorado.edu 